"canal" is a misnomer: the old canal is completely obliterated, and instead a new exit is provided which is almost straight through the abdominal wall, composed of three layers of white fascia, each fitting closely against the walls of the emerging cord.

In some patients it may not be possible, especially in direct hernia, to find anything but a remnant of the transversalis fascia, and in these cases one should mobilize and turn down a flap from the anterior rectus sheath and suture it into Poupart's ligament instead of the transversalis fascia. In small hernias it is unnecessary to get an overlap of the external abdominal aponeurosis ; it is sufficient in these cases to suture a good "bite" of the lower and upper leaves together by interrupted sutures. Gentleness and the avoidance of all unnecessary trauma are strongly recommended, while the use of a gauze wire as a dissecting agent on the cord is deprecated.

As regards suture material I personally favour chromic catgut. The use of wire in any form is not merely unnecessary but may cause serious trouble. First, it is apt to tear the tissues or cut into them, and, secondiy, it acts as a permanent foreign body that may promote sepsis. Kangaroo tendon has the former disadvantage, while silk sutures appear to have the second. I am not aware of any real evidence that catgut is not the best material available.

\section{Causes of Failure}

As to the cause of so many failures in the treatment of inguinal hernia by operation, I submit that they are mainly due to the following: (1) the performance of the Bassini operation; (2) - insufficient and improper suturing at the internal abdominal ring; (3) tearing the edges of Poupart's ligament by means of thick hard catgut or tight ligatures; (4) omitting to warn the patient against any strain or hard work for three months after operation; (5) failure of appreciation on the part of many surgeons of the fact that the operation requires proper planning and careful execution in every detail. It is not to be regarded as an operation "anyone can do with his eyes almost closed," unless he is to "view" his results with his eyes completely closed.

\section{Summary}

- A series of eighty-four cases of inguinal hernia treated by a simple operation, with 100 per cent. success, is published.

No distinction has been made between direct and indirect hernia, as in a few cases notes were incomplete.

No patient admitted under my care during a ten-year period with inguinal hernia was denied operation.

Several patients were admitted to hospital for treatment for other conditions and were found to be suffering from hernia, for which all agreed to have an operation.

The number of patients fitted with a truss by dispensary doctors in recent years appears to be nil. In hospital practice this meant that many of the patients in my list had small hernias, and an overlap of the external oblique aponeurosis was not feasible or practicable at operation.

[Since writing the above I have read the article by Mr. W. Gray in the British Medical Journal of April 6, 1940 , but I have no reason to alter my views on the subject. Perhaps Irish fascia is tougher than that found elsewhere!]

Bibliography

Andrews, Ed. Operative Surgery. Bickham, W. S., 7, 343, Philadelphia

Berne, C. J. (1938). J. Amer. med. Ass., 110, 1812.

Burdick. C. G., and Coley, B. L. (1937). Ann. Surg.. 106. 322

and Higinbotham, N. L. (1935). Ibid. 102, 863 .

Fallis, L. S. (1936). Ibid., 104. 403.

Grace. R. V., and Johnson, V. S. (1937). Ibid., 106, 347

Gray, W. (1940). British Medical Journal, 1, 568

Keynes, G. (1937). Proc. roy. Soc. Med., 30, 529.

Maingot, R. (1937). Postgraduate Surgery, vol. 3, London.

Page, C. M. (1934). British Medical Journal, 2. 896

Porritt. A. E. (1939). Proc. roy. Soc. Med., 32, 893.

\section{SCARLET FEVER IN A PUBLIC SCHOOL}

BY

\section{J. K. BOSTOCK, M.B.}

An epidemic due to the haemolytic streptococcus occurred recently in a public school. Almost every case was swabbed and typed according to the Griffith method, and it was revealed that out of eighty-seven cases of this infection sixty-four were due to Type 18 (Griffith). The remaining twenty-three cases were composed of nine other different Griffith types.

\section{Details of the Epidemic}

Scarlatiniform rashes occurred in five of the sixty-four cases of Type 18 (a proportion of about 1 to 13) and in six of the twenty-three cases composed of various types (a proportion of about 1 in 4). Type 18 was the only one which showed epidemic qualities. It was of fairly low virulence and evinced no tendency to increase in virulence by passage. Complications were few and varied. A proportion of the patients were merely suffering from a nasal discharge, and did not complain of feeling ill.

The school has eight houses-six in a central block and two in separate buildings. The infection was at first confined to one house almost exclusively, eventually spreading slowly through the school. In this particular house twenty-three out of the twenty-four cases were Type 18. In another house eleven cases were all of Type 18. In the six other houses there was a preponderance of Type 18 , but other types were also present. One of these houses had sixteen cases of Type 18 and five other cases each of a different type.

The details of the eighty-seven cases are set out below. With two exceptions-a case of streptococcal pneumonia and one of erysipelas - they were all nasopharyngeal infections.

TABLE I.-Incidence of Scarlatinal Rash among Various Types of Haemolytic Streptococcus

\begin{tabular}{|c|c|c|}
\hline No. of Cases & Type (Griffith) & No. with Rash \\
\hline $\begin{array}{r}3 \\
2 \\
1 \\
1 \\
4 \\
3 \\
1 \\
64 \\
1 \\
1 \\
5 \\
1\end{array}$ & $\begin{array}{rr}1 & \\
2 & \\
3 & \\
5 & \\
12 & \\
13 & \\
17 & \\
18 & \\
20 & \\
28 & \\
\text { Undetermined. } & 3 \text { sterile } \\
\text { swabs ; } 1 \text { not swabbed } \\
\text { Throat swab negative ; close } \\
\text { contact with positive case }\end{array}$ & $\begin{array}{l}\text { 二 } \\
1 \text { case } \\
1 \text { case } \\
5 \text { cases } \\
\text { - } \\
3 \text { cases } \\
\text { (Erysipelas) }\end{array}$ \\
\hline
\end{tabular}

TABLE II.-Analysis of Individual Types, showing Occurrence of Complications, including Rash

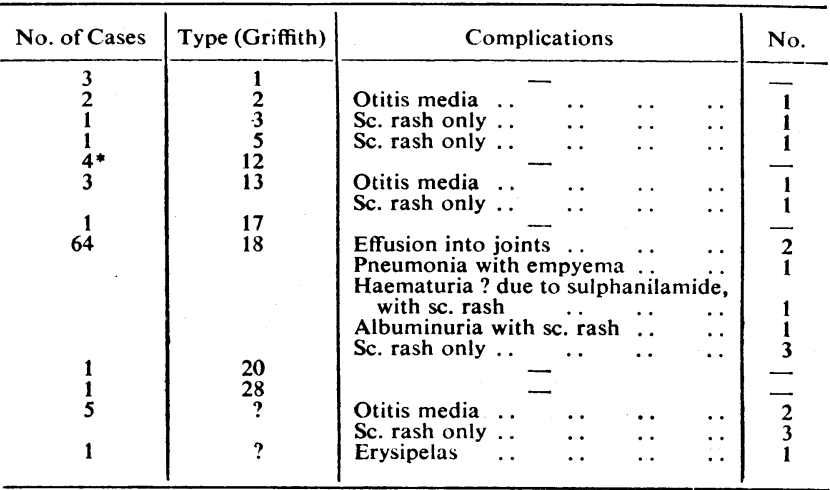

* A typical case of scarlet fever due to this type occurred in the previous term. 


\section{Relapses and Recurrences}

Two cases which demonstrated that immunity to one type did not give immunity to a different type are described below. One case occurred in this epidemic; the other occurred a few years ago, and is included here because it shows the same thing happening in a more dramatic way.

Case 1.-This patient, one of the Type 18 cases of pharyngitis complicated by pain and effusion into the joints, had suffered in the previous term from pharyngitis and infection of the remains of a tonsil due to Type 12 .

Case 2.-A few years before two cases of scarlet fever occurred within four days of each other-one due to Type 13, the other to Type 1. The Type 13 case was mild in character, but had a definite scarlatiniform rash. After about seventeen days both cases were found to be negative to the Dick test and were allowed to convalesce together. Three days later the case due to Type 13 developed a second attack of a more severe character, with intense sore throat and extensive rash. This patient's second swab revealed a Type 1 infection.

In connexion with the above two cases the paragraph below (Stimson, 1936) is of interest :

"Following 1 to 5 per cent. of cases of scarlet fever (the mild ones or those treated with serum, or particularly those with persistently positive Dick tests) relapses may occur from three to six weeks after the onset of the original attack, or recurrences of the disease at a still later period. The new attacks may be milder or more severe than the original one, and, though auto-reinfection is considered possible, are usually due to a new infection. Convalescent cases with persistently positive Dick tests should accordingly be kept separate from other convalescing scarlet fever patients." .

It will be observed that in both these cases the type was different. The Type 12 case contracted a Type 18 infection after a lapse of about two months, and the Type 13 case was infected with Type 1 after barely three weeks. An interesting point is that although the Dick test was negative in the case of Type 13 it was no guarantee against cross-infection by Type 1, which was a more virulent strain. It is also interesting to note that the reverse cross-infection did not take place.

\section{Conclusions}

It seems reasonable to conclude that the production of the rash in this epidemic depended on two factors at least: first, on the individual reaction of the patient to each type and not to any variation in the character of the specific type ; and, secondly, on the rash-producing properties of each individual type, which appeared to be greater in the non-epidemic strains.

Many, if not all, types of haemolytic streptococcus seem to be capable, in certain circumstances, of producing the rash of scarlet fever.

The production of the rash had no effect on the course of the disease, and these cases were almost without complication. It was therefore regarded as of no special significance. Epidemic quality was shown to a high degree in one type only, and the virulence of this infection remained low. Other types presented the usual low degree of infectivity.

A highly specific immunity was apparently set up on two occasions for individual types, as shown by second attacks by dissimilar types at short intervals.

Scarlet fever is a misleading term which suggests a distinction that does not exist between different haemolytic streptococcal infections.

Stimson, P. M. (1936). Common Contagious Diseases, p. 122, London.

\section{USE OF PLASMA OR SERUM AS A SUBSTITUTE FOR WHOLE BLOOD}

BY

\author{
C. H. BEST, M.D.Toronto, D.Sc.Lond., F.R.S. \\ Professor of Physiology, University of Toronto \\ AND
}

D. Y. SOLANDT, M.D.Toronto, Ph.D.Lond. Associate Professor of Physiology, University of Toronto (From the Departments of Physiology and Physiological Hygiene, University of Toronto)

In the issue of the Journal for May 18 (p. 799) we reported the results of the use of concentrated serum in the treatment of traumatic shock in experimental animals. A preliminary account of further work in this field was presented at the Annual Meeting of the American Human Serum Association a few weeks ago.

It is apparent that there is considerable uncertainty over the relative merits of serum and plasma as substitutes for whole blood in the clinic. In our more recent experimental work serum and plasma have been used interchangeably. The results are identical. These materials are employed in experimental and clinical work for two main purposes: first, to provide fluid; and, secondly, to provide the serum proteins, which by their osmotic pressure retain fluid in the blood stream or attract it from the tissues. In so far as these two properties are concerned there is no significant difference between serum and plasma. The amount of fibrinogen in the plasma has a relatively insignificant effect on the production of osmotic pressure. In experimental work sera which produce undesirable reactions are discarded. In our experience most samples of normal or concentrated dog serum produce no undesirable reaction.

\section{The Question of Reactions}

When a substitute for whole blood is used clinically, however, the question of reaction-producing substances is of great importance. It can now be stated that normal serum, concentrated serum, and serum reconstituted after drying in the frozen state have been used in the treatment of many clinical cases without the occurrence of any serious reactions. These points were established by the findings of Thalhimer in New York, Levinson in Chicago, Drury and his collaborators in England, and our clinical colleagues in Toronto. It is true, however, that some groups of investigators reported very troublesome reactions when dried serum was used. These investigators quite naturally prefer dried plasma, and if complete uniformity of product were immediately necessary plasma might well be the choice. In the present circumstances, however, some of those who are preparing dried or concentrated serum and are encountering no serious difficulties may feel that it is unnecessary to make the change. Serum has certain advantages from the viewpoint of preparation, but, on the other hand, plasma is more readily available if material is to be obtained from blood banks.

In the project for supplying blood substitutes for military use, conducted in these departments in collaboration with Drs. Ridout, Chute, and Magladery, concentrated serum has been prepared from some 3,000 blood donors enlisted by the Canadian Red Cross Society. Dried plasma and dried serum will also be made available until a final decision is reached regarding the type or types of blood substitutes best suited to the various conditions under which they may be used. 